\title{
The earliest phases of massive star formation within entire molecular cloud complexes
}

\author{
Frédérique Motte ${ }^{1}$, S. Bontemps ${ }^{2}$, P. Schilke ${ }^{3}$, D. C. Lis ${ }^{4}$, \\ N. Schneider ${ }^{2}$ and K. M. Menten ${ }^{3}$ \\ ${ }^{1}$ AIM, UMR 7158 CEA/CNRS/Paris VII, SAp Bât 709, 91191 Gif-sur-Yvette, France \\ email: motte@cea.fr \\ ${ }^{2}$ Observatoire de Bordeaux, France; \\ ${ }^{3}$ Max-Planck-Institut für Radioastronomie, Germany; \\ ${ }^{4}$ California Institute of Technology, USA
}

\begin{abstract}
We started (sub-)millimeter continuum and line studies of entire molecular cloud complexes located at intermediate distances from the Sun (1-3 kpc). Such an unbiased approach allows to identify and characterize the earliest phases of high-mass stars overlooked by IRAS or $M S X$. Our complete MAMBO-2 surveys of the Cygnus X and NGC 7538 complexes reveal a large population of $\sim 0.1$ pc-size massive young stellar objects (MYSOs) harboring high-mass infrared-quiet protostars. The determination of the nature of all the new millimeter sources is still in progress but we have already collected evidence that the infrared-quiet (or class 0-like) protostellar phase might last as long as the better-known infrared-bright protostellar phase. Besides, our complete census of MYSOs fails to discover the high-mass analogues of pre-stellar dense cores. We propose that the observed lower-density pre-stellar clumps (>1 pc) rapidly concentrate and collapse as also found in the kinematical studies of other prominent clumps. Indeed, $\mathrm{CS}$ and $\mathrm{HCO}^{+}$mappings in W43 and Cygnus X suggest global supersonic contraction with inward velocities of several $\mathrm{km} \mathrm{s}^{-1}$ on parsec scales. Our work and similar studies of entire star-forming complexes will thus definitively contribute to a better knowledge of the earliest phases of high-mass star formation.
\end{abstract}

\section{Background: the earliest phases of high-mass star formation}

High-mass stars, though few in number, play a major role in the energy budget of galaxies. However our current understanding of their formation remains very schematic, especially concerning the earliest phases of the process. High-mass $\left(\mathrm{OB},>8 M_{\odot}\right)$ stars are known to form in dense cores within molecular cloud complexes, by accretion (Yorke \& Sonnhalter 2002; McKee \& Tan 2003) and/or coalescence (e.g. Bonnell et al. 2001). The copious UV flux emitted by a newly-formed central star heats and ionizes its parental molecular cloud leading to the formation and development of a hot core and afterward an $\mathrm{H}$ II region.

In the past few years, the progenitors of ultracompact $\mathrm{H}$ II (UCH II) regions have been searched among bright and red IRAS sources. The criteria generally used aim at selecting high-luminosity sources embedded in a massive envelope and associated with hot gas but no UCH II region (see Kurtz et al. 2000). In this way studies by e.g., Molinari et al. (2000) and Beuther et al. (2002) have identified massive young stellar objects (MYSOs) harboring high-mass infrared-bright protostars. Such surveys are however biased against younger and, thus, probably colder MYSOs which are inconspicuous in the mid-infrared bands of IRAS and $M S X$. These high-mass analogues of low-mass class 0 
protostars and pre-stellar cores (cf. André et al. 2000) are best detected via far-infrared to (sub)millimeter dust continuum and high-density molecular line tracers.

Serendipitous discoveries of these infrared-quiet MYSOs have been made in infrared images ( $I S O / M S X$, revealing a population of so-called IR dark clouds, e.g. Carey et al. 2000; Thompson et al. 2005) and submillimeter continuum maps around well-known IRAS sources (e.g. Molinari et al. 1998; Faúndez et al. 2004; Reid \& Wilson 2005). To make significant progress, one needs to search in a more systematic way for the earliest phases of high-mass star formation. We therefore started (sub-)millimeter continuum and molecular line studies of entire molecular cloud complexes in which high-mass stars are forming (measuring several square degree size images; see e.g. Fig. 1). While having its own merits, this work is also meant to help prepare scheduling and analyzing a key project proposed for the guaranteed time of the SPIRE and PACS instruments aboard the Herschel satellite (Motte, Zavagno, Bontemps et al.).

\section{Unbiased census of MYSOs in high-mass star-forming complexes}

To achieve the spatial resolution of dense cores $(\sim 0.1 \mathrm{pc})$ with current bolometer arrays, it is necessary to select molecular cloud complexes located at distances less than $3 \mathrm{kpc}$. We started surveying Cygnus X, NGC 6334, and NGC 7538 but we hereafter focus on the former high-mass star-forming region which we have studied most extensively. Cygnus X is one of the richest molecular and HiI complexes which is relatively close to the Sun, at $1.7 \mathrm{kpc}$ according to our ${ }^{13} \mathrm{CO}$ study (cf. Schneider et al. 2005). The molecular cloud complex is massive $\left(4 \times 10^{6} M_{\odot}\right.$ over $100 \mathrm{pc}$ diameter $)$, contains one of the largest OB association of our Galaxy (Cyg OB2, cf. Knödlseder 2000) and several well-known MYSOs (e.g. GL2591, S106-IR, DR21, W75N).

\subsection{Wide-field submillimeter continuum imaging}

We used the infrared extinction map we created from 2MASS data (cf. Bontemps et al. in prep.) to select the highest-column density $\left(A_{\mathrm{V}}>15 \mathrm{mag}\right)$ clouds of Cygnus X. The resulting $\sim 3^{\circ^{2}}$ area was imaged in 1999-2003 with the MPIfR bolometer arrays (MAMBO and MAMBO-2) installed at the IRAM $30 \mathrm{~m}$ telescope. Our $1.2 \mathrm{~mm}$ continuum imaging of the Cygnus X molecular cloud complex (Motte et al. 2005, see e.g. Fig. 1) gives a complete view of the cloud structures ranging from 0.09 to $5 \mathrm{pc}$, i.e. from dense cores to clumps (cf. the terminology used for nearby molecular clouds). We performed a multi-resolution analysis to identify a few tens of massive large-scale $(>1 \mathrm{pc})$ clumps and extract 131 compact $(\sim 0.1 \mathrm{pc})$ dense cores. The masses of the latter sources range from 4 to $950 M_{\odot}$ assuming a dust temperature of $20 \mathrm{~K}$ and a dust mass opacity of $0.01 \mathrm{~cm}^{2} \mathrm{~g}^{-1}$. Their mean densities vary from $\sim 10^{5}$ to $\sim 10^{7} \mathrm{~cm}^{-3}$, which is 1 to 100 times larger than those of low-mass dense cores averaged over $\sim 0.1 \mathrm{pc}$ (e.g. Ward-Thompson et al. 1999). They thus represent good candidate sites for forming intermediate- to high-mass stars and will hereafter be called MYSOs.

\subsection{Evolutionary state of the Cygnus X MYSOs}

We have used existing surveys and started various follow-up studies to determine the exact nature of each of these new MYSOs: pre-stellar dense core, infrared-quiet protostar, infrared-bright protostar, or UCH II region. Preliminary results are given below:

- Most $(90 \%)$ of the millimeter sources are new and do not exhibit centimeter-wavelength radio emission, which generally is the signature of an already developed H II region. A large fraction of the Cygnus X MYSOs are excellent candidates for being at younger evolutionary stages. 


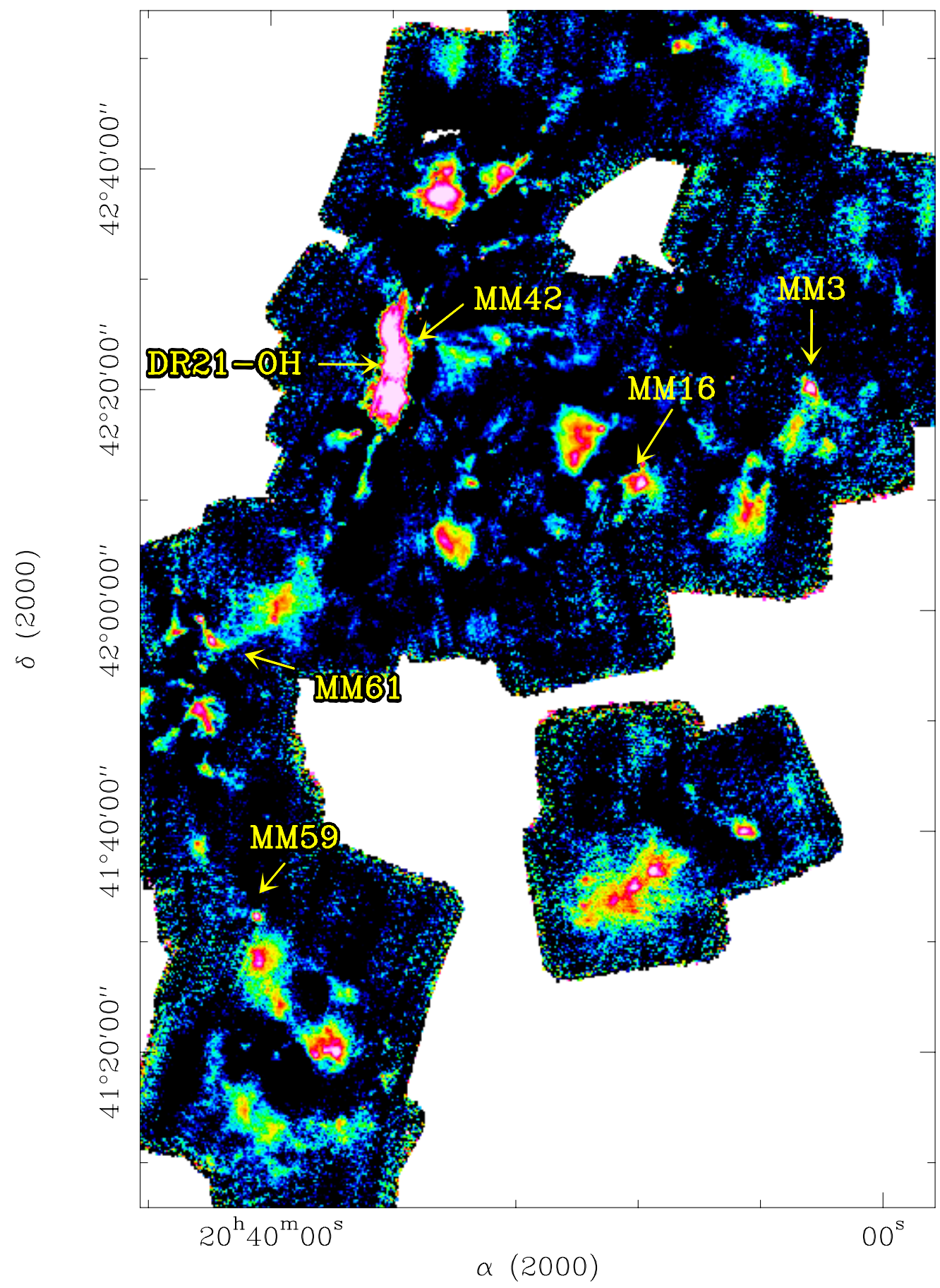

Figure 1. $1.2 \mathrm{~mm}$ imaging of the main part of the Cygnus X molecular cloud complex (Motte, Bontemps, Schilke, et al. 2005). Seventy-two MYSOs candidates (compact dense cores with $\sim 0.1$ pc diameters) and $\sim 20$ more diffuse clumps $(>1 \mathrm{pc})$ were discovered in this $1.5^{\circ^{2}}$ mosaic. The best candidates for young high-mass protostars we currently identified (i.e. IR-quiet MYSOs that exhibit powerful outflows and in some cases infall signatures) are labelled. 
- Careful inspection of the $M S X$ images shows that one third of the millimeter sources are associated with mid-IR emission and another one third are seen in absorption against the infrared background. The first category of MYSOs may thus be infrared-bright protostars while the others may either be infrared-quiet (class 0-like) protostars or pre-stellar dense cores. Note that the infrared-quiet MYSOs are associated with neither more massive nor less massive dense cores than infrared-bright MYSOs. We thus confirm that there probably is an evolutionary trend from infrared-quiet to infrared-bright sources.

- The molecular line survey we recently conducted at the IRAM $30 \mathrm{~m}$ telescope for the most massive $\left(>40 M_{\odot}\right)$ millimeter sources of Cygnus X shows that they all drive powerful outflows. Some of them also exhibit hot core, maser, and infall signatures. If these preliminary findings are confirmed for our complete sample of MYSOs, the vast majority $(80 \%)$ of the dense cores discovered with MAMBO-2 may be high-mass protostars while very few seem to be be pre-stellar dense cores.

- We embarked on various other follow-up observations with the IRAM 30 m, Effelsberg, CSO, and FCRAO radiotelescopes as well as with the IRAM and VLA interferometers. A particular emphasis is given to studies looking for signatures of outflow, infall, hot cores, and masers (see e.g. Sect. 3).

\section{Large-scale dynamical inflow toward infrared-quiet MYSOs}

In an effort to constrain the kinematics of the earliest phases of high-mass star formation, we have selected the most promising infrared-quiet MYSOs identified by our (sub)millimeter surveys (see e.g. Fig. 1). We started with sources in the Cygnus X and W43 molecular cloud complexes, W43 being a more extreme case of a high-mass starforming region and located further than Cygnus X (5.5 kpc, see Motte et al. 2003). We used the IRAM $30 \mathrm{~m}$ and CSO $10 \mathrm{~m}$ telescopes to make maps in the $(2-1),(3-2)$, and (5-4) transitions of $\mathrm{CS}$ and $\mathrm{C}^{34} \mathrm{~S}$, and in the (3-2) transition of $\mathrm{HCO}^{+}$and $\mathrm{H}^{13} \mathrm{CO}^{+}$. Strikingly, most of the $\mathrm{CS}$ and/or $\mathrm{HCO}^{+}$lines, which look optically thick, show a deep broad self-absorption which is redshifted relative to the systemic velocity marked by optically thin lines (see e.g. Fig. 2). Such line profiles (stronger blueshifted emission and/or redshifted self-absorption) are classical signatures of the gravitational collapse observed toward low-mass class 0 protostars (e.g. Gregersen et al. 1997).

However, our maps suggest that these inward motions are not associated with local infall toward individual high-mass protostars but resemble a more global contraction acting on parsec scales (cf. Fig. 3). Using a radiative transfer code (cf. Belloche et al. 2002) and a source model constrained by our mm continuum and molecular lines maps, we are inferring constant inward velocities of $1-2 \mathrm{~km} \mathrm{~s}^{-1}$ for ten MYSOs in Cygnus X and W43. These dynamical inflows are one order of magnitude faster and one order of magnitude larger-scale than the quasi-static global contraction often observed for lowmass pre-stellar cores (e.g. Lee et al. 2001).

Interestingly enough, these supersonic inward motions are only observed in the densest parts of the complexes: the DR21 filament and the most prominent infrared-quiet MYSOs of W43 and Cygnus X. These may be privileged sites and the only MYSOs of these complexes that are undergoing a global supersonic contraction. But since the $\mathrm{HCO}^{+}$and CS lines are generally not self-absorbed toward the other MYSOs, it is also possible that these tracers are not optically thick enough to show inflow signatures. Other authors mentioned similar overall collapses for parsec sections of the W51 and NGC 2264 molecular cloud complexes (Rudolph et al. 1990; Williams \& Garland 2002; Peretto et al. submitted, see also Wu et al. this volume). 


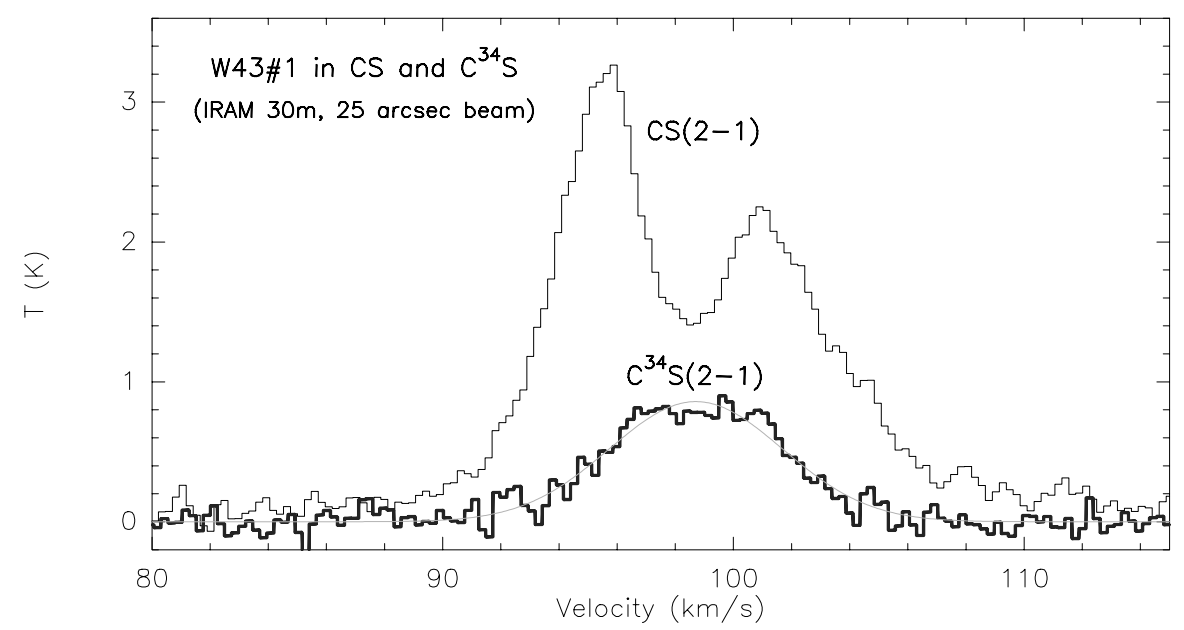

Figure 2. $\mathrm{CS}$ and $\mathrm{C}^{34} \mathrm{~S}(2-1)$ spectra toward W43-MM1 suggesting a $\sim 2 \mathrm{~km} / \mathrm{s}$ inflow.

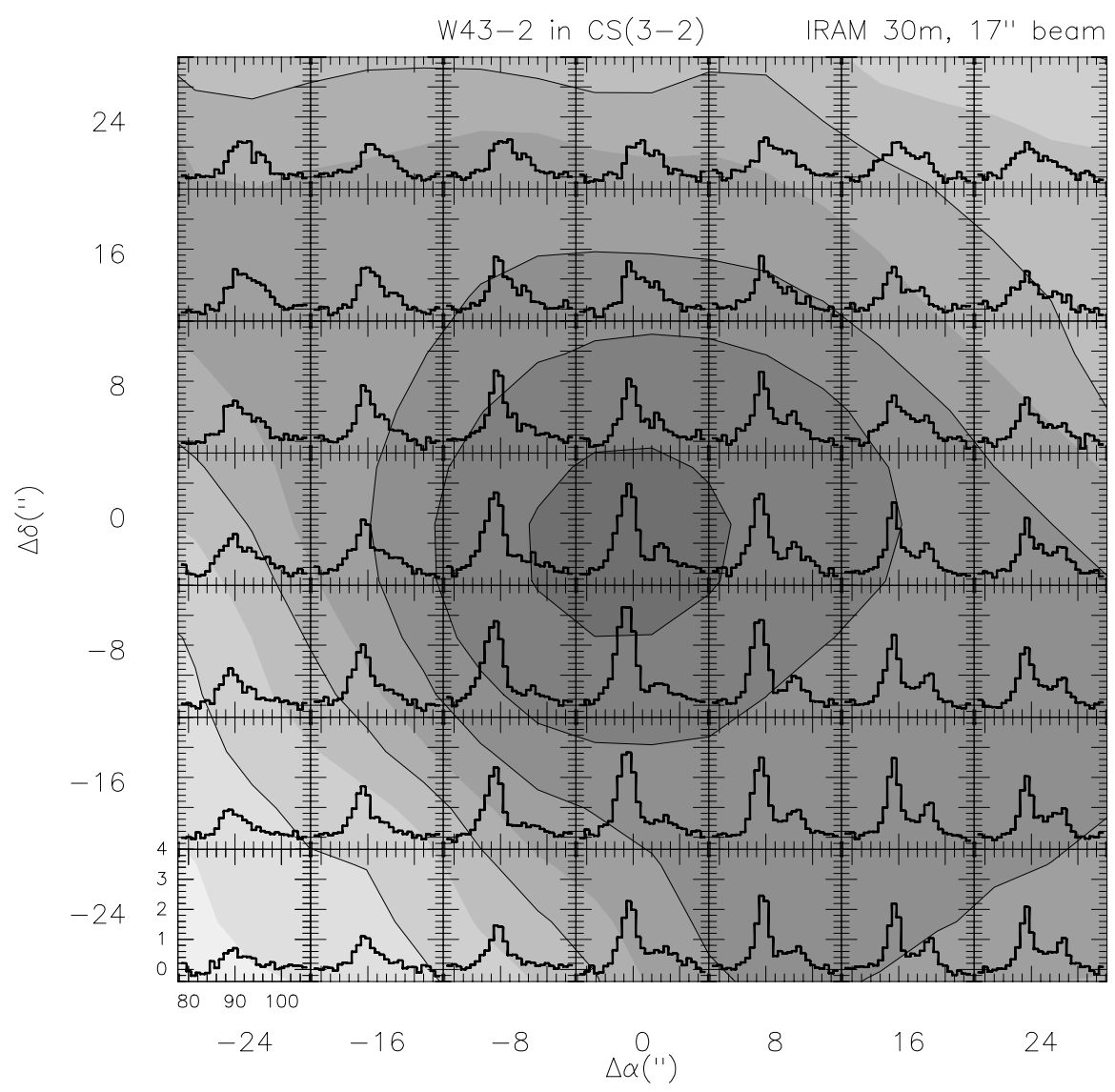

Figure 3. Grid of CS(3-2) spectra (thick histograms) observed around the infrared-quiet MYSO W43-MM2 and plotted against its MAMBO map (gray scale and thin contours). Data for both tracers were obtained with the IRAM $30 \mathrm{~m}$ telescope with $17^{\prime \prime}$ and $11^{\prime \prime}$ resolution, respectively. Since independent spectra separated by up to $\sim 40^{\prime \prime}$ show similar redshifted self-absorption, this mapping suggests global contraction on at least 1 pc scales. 


\section{Summary and preliminary conclusions}

We have begun long-term studies of entire molecular cloud complexes, for which a summary and preliminary conclusions are given below:

- Our (sub-)millimeter continuum and line surveys provide unbiased and comprehensive samples of $\sim 0.1 \mathrm{pc}$ MYSOs at evolutionary stages from infrared-quiet sources to UCH IIs. In particular, the complete sample of Cygnus X MYSOs confirms the existence of a large population of massive (class 0-like) protostars overlooked by $I R A S$ and $M S X$.

- Given the relative number of infrared-quiet to infrared-bright protostars found in Cygnus $\mathrm{X}$, the infrared-quiet protostellar phase (high-mass analogue of the low-mass class 0 phase) may last as long as that of infrared-bright MYSOs.

- Our kinematics surveys show that the most prominent parts of the W43 and Cygnus X cloud complexes are undergoing global supersonic contraction: $1-2 \mathrm{~km} \mathrm{~s}^{-1}$ inflow velocities over $>1$ pc. If these inward motions continue at smaller scales high-mass protostars could accrete at a rate as high as $10^{-3} M_{\odot} \mathrm{yr}^{-1}$.

- The fact that we did not find any clear case of a pre-stellar core in Cygnus X suggests that these are short-lived cloud structures. They may simply not exist if highmass protostars are all formed via coalescence of intermediate-mass protostars. However, our kinematical study favors a scenario where more diffuse pre-stellar clumps (like the starless cloud structures identified in Fig. 1 with $\sim 1 \mathrm{pc}$ and $10^{4} \mathrm{~cm}^{-3}$ ) are compressed and thus rapidly collapse to high-mass protostars.

\section{References}

André, P., Ward-Thompson, D., Barsony, M. 2000, in: V. Mannings, A. Boss, \& S. Russell (ed.), Protostars \& Planets IV, 59

Belloche, A., André, P., Despois, D., Blinder, S. 2002, A\&̛A, 393, 927

Beuther, H., Schilke, P., Menten, K. M., Motte, F., Sridharan, T. K., Wyrowski, F. 2002, ApJ, 566,945

Bonnell, I. A., Bate, M. R., Clarke, C. J., Pringle, J. E. 2001, MNRAS, 323, 794

Carey, S. J., Feldman, P. A., Redman, R. O., Egan, M. P., MacLeod, J. M., Price, S. D. 2000, ApJ, 543, L157

Chandler, C. J., Moore, T. J. T., Mountain, C. M., Yamashita, T. 1993, MNRAS, 261, 694

Faúndez, S., Bronfman, L., Garay, G., Chini, R., Nyman, L.-Å., May, J. 2004, A\&A, 426, 97

Gregersen, E. M., Evans, N. J. II, Zhou, S., Choi, M. 1997, ApJ, 484, 256

Knödlseder, J. 2000, A\& $A$, 360, 539

Kurtz, S., Cesaroni, R., Churchwell, E., Hofner, P., Walmsley, C. M. 2000, in: V. Mannings, A. Boss, \& S. Russell (ed.), Protostars \& Planets IV, p. 299

Lee, C. W., Myers, P. C., Tafalla, M. 2001, ApJS, 136, 703

McKee, C. F., Tan, J. C. 2003, ApJ, 585, 850

Molinari, S., Brand, J., Cesaroni, R., Palla, F. 2000, A\&A, 355, 617

Molinari, S., Testi, L., Brand, J., Cesaroni, R., Palla, F. 1998, ApJ, 505, L39

Motte, F., Schilke, P., Lis, D. C. 2003, ApJ, 582, 277

Motte, F., Bontemps, S., Schilke, P., Menten, K. M., Schneider, N., Broguière, D., in prep.

Reid, M. A.; Wilson, C. D. 2005, ApJ, 625, 891

Rudolph, A., Welch, W. J., Palmer, P., Dubrulle, B. 1990, ApJ, 363, 528

Schneider, N., Bontemps, S., Simon, R., Jakob, R., Motte, F., Miller, M. 2005, in prep.

Thompson, M. A., Gibb, A. G., Hatchell, J. H., Wyrowski, F., Pillai, T. 2005, in: A. Wilson (ed.), The dusty and molecular universe: a prelude to Herschel and ALMA, ESA SP-577, p. 425

Ward-Thompson, D., Motte, F., André, P. 1999, MNRAS, 305, 143

Williams, J. P., Garland, C. A. 2002, ApJ, 568, 259

Yorke, H. W., Sonnhalter, C. 2002, ApJ, 569, 846 